\title{
A Data-driven Minimum Stiffness Prediction Method for Machining Regions of Aircraft Structural Parts
}

\section{Jiarui Chen}

Nanjing University of Aeronautics and Astronautics

Yingguang Li ( $\sim$ liyingguang@nuaa.edu.cn )

Nanjing University of Aeronautics and Astronautics

Xu Liu

Nanjing University of Aeronautics and Astronautics

Tianchi Deng

Nanjing University of Aeronautics and Astronautics

\section{Research Article}

Keywords: Aircraft structural parts, Stiffness, Surrogate models, Graph neural networks

Posted Date: November 30th, 2021

DOI: https://doi.org/10.21203/rs.3.rs-1103833/v1

License: (9) This work is licensed under a Creative Commons Attribution 4.0 International License. Read Full License

Version of Record: A version of this preprint was published at The International Journal of Advanced Manufacturing Technology on March 7th, 2022. See the published version at https://doi.org/10.1007/s00170-022-08991-x. 


\title{
A Data-driven Minimum Stiffness Prediction Method for Machining Regions of Aircraft Structural Parts
}

\author{
Jiarui Chen ${ }^{1}$, Yingguang $\mathrm{Li}^{1, *}, \mathrm{Xu} \mathrm{Liu}^{2}$, Tianchi Deng ${ }^{1}$ \\ *Corresponding author: liyingguang@nuaa.edu.cn \\ ${ }^{1}$ College of Mechanical and Electrical Engineering, Nanjing University of Aeronautics and Astronautics, Nanjing, China \\ ${ }^{2}$ School of Mechanical and Power Engineering, Nanjing Tech University, Nanjing, China
}

\begin{abstract}
Large thin-walled structural parts have been widely used in aircrafts for the purpose of weight reduction. These parts usually contain various thin-walled complex structures with weak local stiffness, which are easy to deform during machining if improper cutting parameters are selected. Thus, local stiffness has to be seriously considered during the machining parameter planning. Existing stiffness calculation methods mainly include mechanics calculation methods, empirical formula methods, finite element methods, and surrogate-based methods. However, due to the structural complexity, these methods are either inaccurate or time consuming. To address this issue, this paper proposes a data-driven method for stiffness prediction of aircraft structural parts. First, machining regions of aircraft structural part finishing are classified into bottom, sidewall, rib and corner to further define the minimum stiffness of machining regions. Then, by representing the part geometry with attribute graph as the input feature, while computing the minimum stiffness using FEM as the output label, stiffness prediction is turned to a graph learning task. Thus, a graph neural network (GNN) is designed and trained to map the attribute graph of a machining region to its minimum stiffness. In the case study, a dataset of aircraft structural parts is used to train four GNN models to predict the minimum stiffness of the defined four types of machining regions. Compared with FEM results, the average percentage errors on the test set are $6.717 \%, 7.367 \%, 7.432 \%$ and $5.962 \%$ respectively. In addition, the data driven model once trained, can greatly reduce the time in predicting the stiffness of a new part compared with FEM, which indicates that the proposed method can meet the engineering requirements in both accuracy and computational efficiency.
\end{abstract}

Keywords: Aircraft structural parts; Stiffness; Surrogate models; Graph neural networks 


\section{Introduction}

To improve flight performance, large thin-walled structural parts have been widely used in various aircrafts [1]. These parts are of lightweight but usually complex structures as well as weak local stiffness. During the finishing process of this kind of parts, elastic deformation and dimensional error appear easily in the regions with weak stiffness due to cutting forces caused by improper machining parameters [2] [3], as shown in Fig. 1. Therefore, local stiffness should be seriously considered in parameter selection.

Traditional stiffness calculation methods mainly include mechanics calculation methods [4], empirical formula methods [5], finite element methods (FEM) [6] and surrogate-based methods [7]. However, due to the structural complexity, these methods are either inaccurate or time consuming, which is far away from the requirements of real manufacturing. As a consequence, machining parameters now are still mainly determined by technologists, and to avoid elastic deformation and dimensional error, conservative machining parameters are usually selected which will greatly reduce the production efficiency [3].
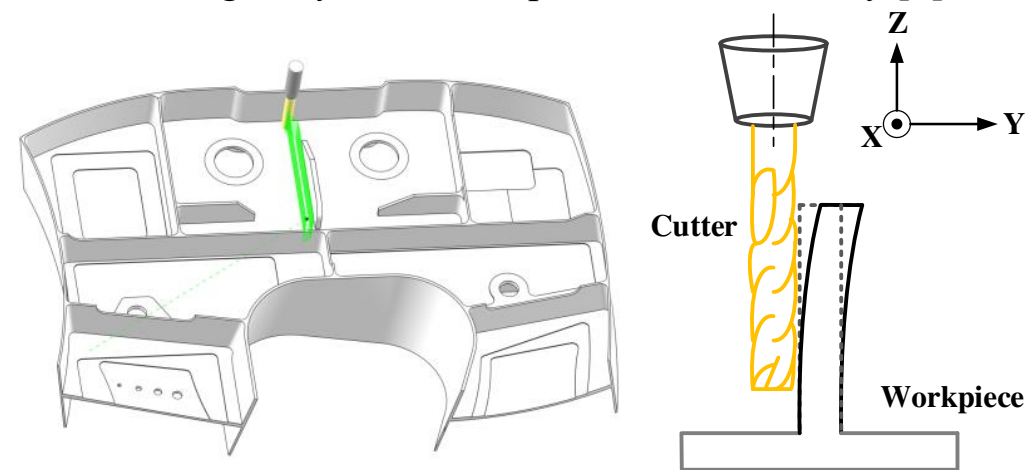

Fig. 1 Local elastic deformation of the part.

This paper proposes a data-driven method for stiffness prediction of aircraft structural parts to support automatic machining parameter planning. First, machining regions of aircraft structural part finishing are classified into bottom, sidewall, rib and corner for further definition of minimum stiffness. By representing the part geometry with attribute graph as the input feature, while computing the minimum stiffness using FEM as the output label, stiffness prediction is turned to a graph learning task. A graph neural network (GNN) is then designed and trained to map the attribute graph of a machining region to its minimum stiffness. In the case study, a dataset of aircraft structural parts is used to train the GNN model for predicting the minimum stiffness of four types of typical machining regions, the average percentage errors compared with FEM are $6.717 \%, 7.367 \%, 7.432 \%$ and 5.962\% respectively, which indicates that the proposed method can meet the engineering requirements. In addition, the data driven model once trained, can greatly improves the computational efficiency in predicting stiffness compared with the FEM.

\section{Related Works}

The stiffness describes the ability of a structure to resist elastic deformation. As the structures in different regions of a part usually have different stiffness, it is of great significance to calculate the stiffness in different regions for selecting machining parameters. Existing stiffness calculation methods can be generally classified into mechanics calculation methods, empirical formula methods, FEM and surrogate-based methods.

\subsection{Mechanics calculation methods}

The mechanics calculation methods approximate the structure of parts as thin-walled 
plate or beam, and calculate their local stiffness based on the thin-walled plate theory or beam theory, which can be used to further analyze the part deformation. For the use of thin-walled plate theory, Tang et al. [8] calculated the stiffness of parts for constructing a static deformation prediction model under different linear loads and thicknesses. Wu et al. [9] integrated the finite difference method into the calculation process of thin-walled plate theory to establish a machining deformation prediction model which is suitable for different machining parameters. Gao et al. [10] built a semi-analytical model of thin-walled parts to analyze the influence of initial residual stress and stiffness on machining deformation. For the beam theory, Liu et al. [11] and Jia et al. [4] simplified the sidewall of parts to cantilever beam, simply supported beam, etc., which realized the machining deformation prediction under different cutting forces.

The above mechanics calculation methods have a mature theoretical system and can obtain accurate results under ideal circumstances. However, to consider a real part with complex structures, these methods have to induce a lot of simplifications and approximations, which will make the inaccurate stiffness prediction result.

\subsection{Empirical formula methods}

The empirical formula methods first choose the most sensitive factors of stiffness to establish empirical models, and then carry out a parameter identification operation using experimental data to obtain the determinate models. Zhang et al. [12] built an empirical model which considered elastic modulus, thickness and outer diameter of the part to calculate the stiffness of any point on the thin-walled cylindrical part. On this basis, Wang [13] used this model to calculate the stiffness of the working cylinder in the pump-controlled hydraulic press system. Ma et al. [5] constructed a cutting unit based on cutter contact point with various cutting widths, and then established a calculation model to analyze the stiffness variation at different cutter contact points in NC machining.

The empirical formula methods directly calculate the stiffness of the part, and is fast enough in real manufacturing. However, empirical models are usually only applicable for a specific set of parts. Moreover, it is really difficult to build an empirical model for calculating the stiffness of aircraft structural parts with complex structures.

\subsection{Finite element methods}

FEM establishes finite element models of the part and use professional CAE software to obtain the stiffness information. Wan et al. [14] and Liu et al. [15] obtained the element stiffness matrix of a part based on finite element simulation (FES) for predicting the dimensional error in machining the part surface. Wang et al. [6] calculated the local stiffness of large thin-walled parts based on FES for proposing a real-time compensation method for machining errors. Smith et al. [16] introduced a force placed at different points in the local region of a part and calculated the displacement of these points based on FES, so as to obtain the minimum stiffness of this region. Huang et al. [17] proposed an equivalent bending strain energy method based on FES, which was used to analyze the stiffness variations in machining.

The results of FEM are more accurate than other methods. However, its computational cost is extremely high, e.g., it takes several hours for the stiffness calculation of an aircraft structural part with small size, while for the large parts, it usually takes more than one day which is unacceptable in real industry.

\subsection{Surrogate-based methods}

Because FEM are computational expensive, some scholars use the data of FEM to build a surrogate model to perform approximate stiffness calculation [18] [19]. This class of existing methods mainly choose neural networks as the surrogate model to predict the element stiffness 
matrix of a part, which is used to analyze and optimize the part structure based on the loading condition. Sun et al. [20] and Jia et al. [21] took the relative coordinates of grid nodes as the sample features, and then built a three-layered neural network and a convolutional neural network respectively to predict the element stiffness matrix of a part. White et al. [22] established a two-layered neural network model to map the geometrical parameters of microscale metamaterials to the element stiffness matrix. Qian et al. [7] improved the above method and built a dual-model neural network to improve the accuracy of topology optimization.

Existing surrogate models are mainly oriented to the structural design of parts, where the stiffness matrix and stress matrix are used to solve the strain of a part, which can't be directly used to calculate the local stiffness. In addition, due to the difficulty to directly vectorize the local region for machine learning models, existing studies only select the size, the type of grid elements, and the coordinates of grid nodes as the sample features, which could be regarded as a big simplification to the prediction task.

Although the above researches have achieved great progress for the stiffness calculation, there are still some shortcomings about the accuracy or computational efficiency for aircraft structural parts. Therefore, this paper proposes a data driven method for predicting the local minimum stiffness of a part. The prediction is converted to a graph learning task by representing the part geometry as an attribute graph. The stiffness calculated by FEM is used as the labels and a graph neural network (GNN) is designed to establish the map between the attribute graph of a machining region and its local minimum stiffness. The rest of this paper is organized as follows. Section 3 will calculate the minimum stiffness of machining regions based on FEM and the proposed method for predicting it quickly and approximately. Section 4 will establish a dataset of aircraft structural parts for training and testing the proposed models. Section 5 will describe the conclusion and future work.

\section{Method}

For the proposed data-driven stiffness prediction method, machining regions of aircraft structural part finishing is first classified into bottom, sidewall, rib and corner for further definition of minimum stiffness. Then, with the minimum stiffness calculated by FEM as the

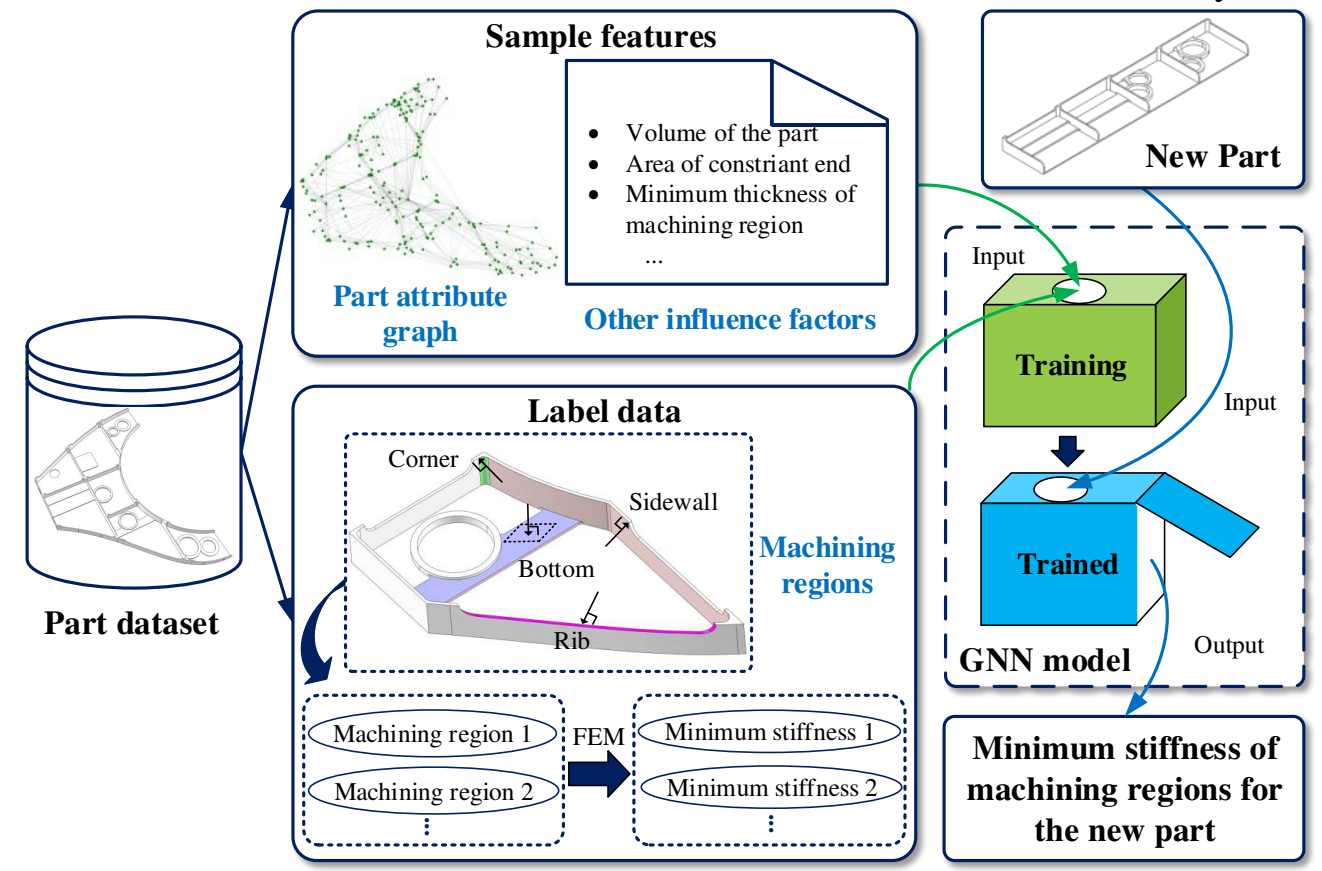

Fig. 2 The workflow of the proposed method. 
output label, and the part model represented by attribute graph as the input feature, the stiffness prediction task is turned to a graph learning task. Next, a graph neural network (GNN) is designed and trained to map the attribute graph of a machining region to its minimum stiffness. The workflow of the proposed method is shown in Fig. 2.

\subsection{FEM-based minimum stiffness calculation of machining regions}

The NC process plan of aircraft structural parts is composed of a large number of ordered machining operations, and each machining operation corresponds to a machining region, whose minimum stiffness is significant to machining parameter selection of the machining operation. To calculate the minimum stiffness of the machining region, the machining regions in aircraft structural part finishing are first classified into bottom, sidewall, rib and corner. Next, the minimum stiffness of each type of machining region is defined, and then calculated by FEM, which will be taken as the output label of the data-driven model.

\subsubsection{The definition of minimum stiffness}

In the NC process planning of aircraft structural parts finishing, technologists usually group the machining regions in bottom, sidewall, rib and corner, which is based on the structural and positional similarity. Different types of machining regions vary in cutting position, cutting force, and constraint condition. For each type of the machining region, the minimum stiffness direction corresponding to a point can be intuitively determined as the material thickness direction of the part, which is further validated by FEM. Thus, the minimum stiffness direction in the bottom region is perpendicular to the bottom face, the minimum stiffness direction in the sidewall region, rib region, and corner region is perpendicular to the face of the side wall, as shown in Fig. 3.

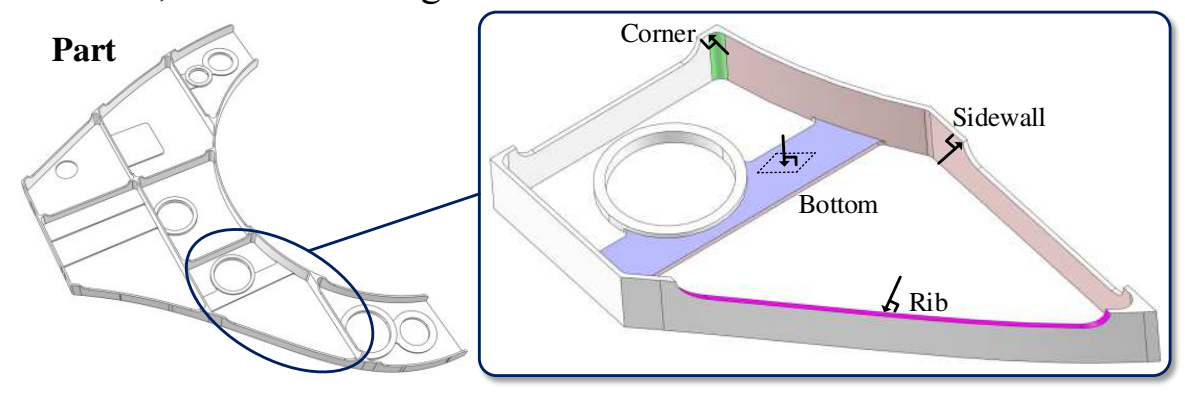

Fig. 3 The minimum stiffness direction of four types of typical machining regions.

After the minimum stiffness direction is determined for each type of machining region, the minimum stiffness can be further defined. For a machining region, it is first divided into multiple discrete grid elements after meshing, including $p$ nodes. Then, the stiffness of each node in the minimum stiffness direction is calculated and represented as $k_{1}, k_{2}, \ldots, k_{p}$. Next, the minimum stiffness $k_{\min }$ of the region is defined as the minimum value of $k_{1}, k_{2}, \ldots, k_{p}$, i.e., $k_{\min }:=\min \left(k_{1}, k_{2}, \ldots, k_{p}\right)$. In addition, for the corner region, the edge points with thinnest thickness always reach the lowest stiffness, thus to improve the efficiency, only the points at the corner edge is considered in the minimum stiffness calculation.

\subsubsection{The calculation of minimum stiffness}

The minimum stiffness of each machining region can be calculated based on FEM. First, the part is divided into grid elements with a large quantity of nodes after meshing. The minimum stiffness direction of all the nodes is calculated according to the associated machining regions. Then, based on the calculated minimum stiffness directions, multiple coordinate systems, which have the same origin but differ in coordinate directions, are built to 
divide the nodes into multiple groups. Each group corresponds to a coordinate system whose coordinate directions are parallel to the minimum stiffness directions corresponding to the nodes in the group. Since the stiffness matrix in FEM only records the information in coordinate directions, it is required to construct new coordinate systems according to the required directions, i.e., minimum stiffness directions.

For each group of nodes, FEM is used to calculate the stiffness in the minimum stiffness direction, which contains five steps. First, all the element stiffness matrices in the global coordinate system are obtained. Secondly, coordinate system transformation is used to obtain the element stiffness matrices in the coordinate system corresponding to the group. Thirdly, the global stiffness matrix is combined based on the element stiffness matrices, where the large values are used to deal with boundary conditions. Fourthly, the global compliance matrix is calculated based on the global stiffness matrix, and the stiffness of all the nodes is further calculated. Fifthly, the stiffness of the nodes in the group is screened out from all the node stiffness. After obtaining the stiffness of all nodes in their respective directions, the minimum stiffness of each machining region is searched based on node-region affiliations. The process is shown in Fig. 4, and the detailed calculation process of node stiffness in each group is as follows.

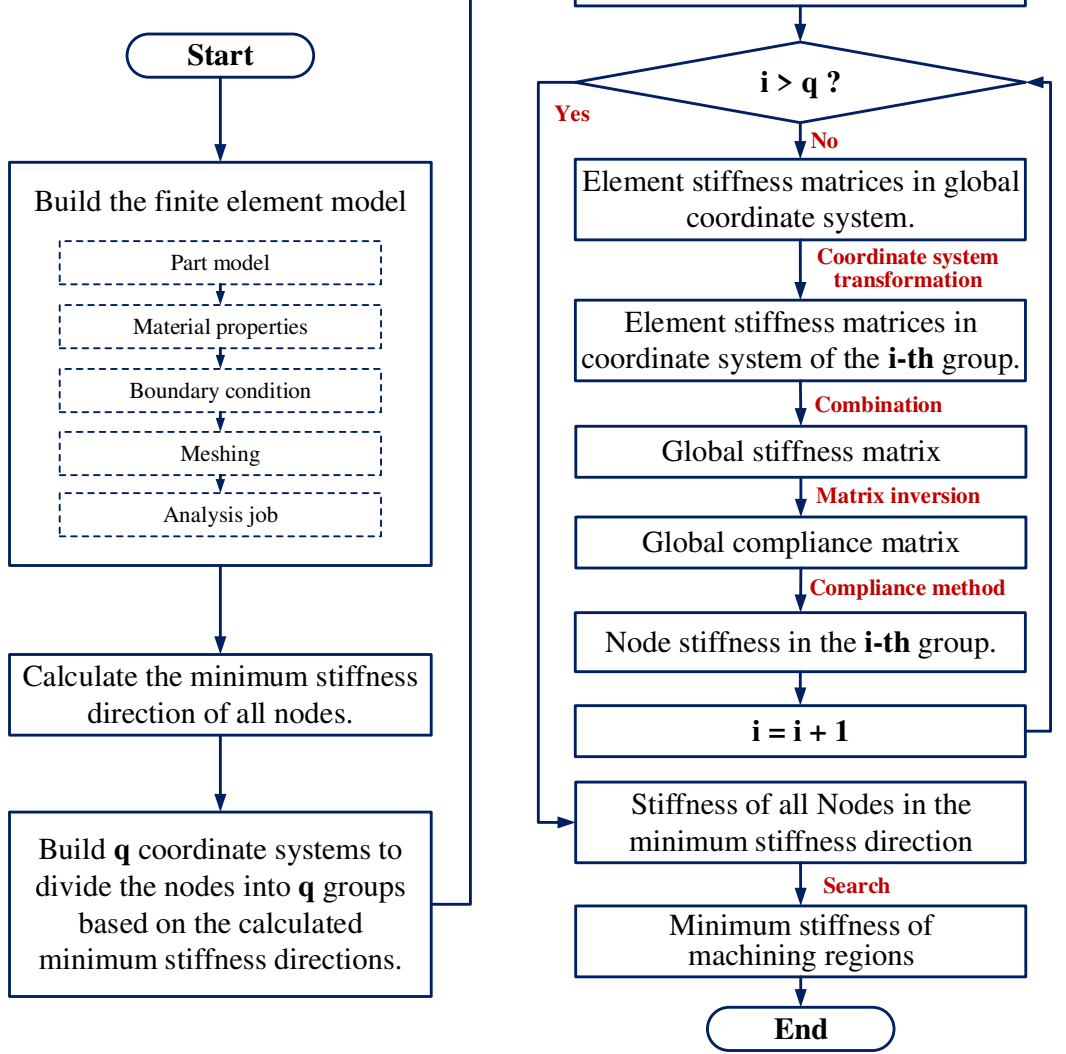

Fig. 4 The calculation process for the minimum stiffness of machining regions.

For each group, there is a local coordinate system whose coordinate directions are parallel to the minimum stiffness directions corresponding to the nodes in the group. It is first required to obtain all element stiffness matrices in global coordinate system, whose generalized definition is expressed as follows:

$$
\mathbf{F}_{e}=\mathbf{D}_{e} \mathbf{U}_{e}
$$

Where $\mathbf{D}_{e} \in \mathbb{R}^{3 n^{\prime} \times 3 n^{\prime}}, \mathbf{F}_{e} \in \mathbb{R}^{3 n^{\prime} \times 1}$, and $\mathbf{U}_{e} \in \mathbb{R}^{3 n^{\prime} \times 1}$ are the element stiffness matrix, element load matrix, and element displacement matrix respectively, $n^{\prime}$ represents the number 
of nodes contained in the grid element.

The coordinate directions of the global coordinate system may not be parallel to the minimum stiffness directions of the nodes in the group, yet the stiffness matrix only records the information in the coordinate directions. Thus, it is required to transform the global coordinate system to the local coordinate system whose coordinate directions are parallel to the minimum stiffness directions that corresponds to the nodes in the group. Let $\mathbf{R}_{e} \in$ $\mathbb{R}^{3 n^{\prime} \times 3 n^{\prime}}$ be the rotation matrix from global coordinate system to the new coordinate system, based on the theory of structural mechanics, the coordinate transformation of $\mathbf{D}_{e}$ is realized by first transforming the element load matrix $\mathbf{F}_{e}^{\prime}$ and the element displacement matrix $\mathbf{U}_{e}^{\prime}$, i.e., $\mathbf{F}_{e}^{\prime}=\mathbf{R}_{e} \mathbf{F}_{e}$ and $\mathbf{U}_{e}^{\prime}=\mathbf{R}_{e} \mathbf{U}_{e}$. Then the new element stiffness matrix $\mathbf{D}_{e}^{\prime}$ can be calculated based on $\mathbf{F}_{e}^{\prime}$ and $\mathbf{U}_{e}^{\prime}$, i.e., $\mathbf{F}_{e}^{\prime}=\mathbf{D}_{e}^{\prime} \mathbf{U}_{e}^{\prime}$. Thus, the relation between $\mathbf{D}_{e}^{\prime}$ and $\mathbf{D}_{e}$ is as follow:

$$
\mathbf{D}_{e}^{\prime}=\mathbf{R}_{e} \mathbf{D}_{e} \mathbf{R}_{e}^{-1}
$$

Where, matrix $\mathbf{R}_{e}$ is an orthogonal matrix which satisfies $\mathbf{R}_{\mathrm{e}}^{-1}=\mathbf{R}_{e}^{\top}$, $\mathrm{T}$ represents matrix transposition, thus $\mathbf{D}_{e}^{\prime}$ can be efficiently calculated by:

$$
\mathbf{D}_{e}^{\prime}=\mathbf{R}_{e} \mathbf{D}_{e} \mathbf{R}_{e}^{\top}
$$

Then, the global stiffness matrix $\mathbf{D}^{\prime} \in \mathbb{R}^{3 n \times 3 n}$ in the coordinate system corresponding to the group is combined based on all element stiffness matrices, where $n$ is the total number of nodes. To deal with the boundary conditions, the global stiffness matrix elements, which corresponds to the nodes at the constraint end, are substituted by the large values. Next, compliance method is used to calculate the node stiffness. Through the inverse of $\mathbf{D}^{\prime}$, the global compliance matrix $\mathbf{C}^{\prime} \in \mathbb{R}^{3 n \times 3 n}$ is obtained, whose diagonal elements are used to calculate the node stiffness in the coordinate directions. More detailed descriptions are as follow:

$\mathbf{C}^{\prime}$ is the inverse matrix of $\mathbf{D}^{\prime}$ in the coordinate system corresponding to the group, it satisfies $\mathbf{U}^{\prime}=\mathbf{C}^{\prime} \mathbf{F}^{\prime}$, which can be expanded as:

$$
\left[\begin{array}{c}
u_{1 x} \\
u_{1 y} \\
u_{1 z} \\
\vdots \\
u_{n x} \\
u_{n y} \\
u_{n z}
\end{array}\right]=\left[\begin{array}{ccccccc}
c_{11} & c_{12} & c_{13} & \ldots & c_{1,3 n-2} & c_{1,3 n-1} & c_{1,3 n} \\
c_{21} & c_{22} & c_{23} & \ldots & c_{2,3 n-2} & c_{2,3 n-1} & c_{2,3 n} \\
c_{31} & c_{32} & c_{33} & \ldots & c_{3,3 n-2} & c_{3,3 n-1} & c_{3,3 n} \\
\vdots & \vdots & \vdots & \ddots & \vdots & \vdots & \vdots \\
c_{3 n-2,1} & c_{3 n-2,2} & c_{3 n-2,3} & \ldots & c_{3 n-2,3 n-2} & c_{3 n-2,3 n-1} & c_{3 n-2,3 n} \\
c_{3 n-1,1} & c_{3 n-1,2} & c_{3 n-1,3} & \ldots & c_{3 n-1,3 n-2} & c_{3 n-1,3 n-1} & c_{3 n-1,3 n} \\
c_{3 n, 1} & c_{3 n, 2} & c_{3 n, 3} & \ldots & c_{3 n, 3 n-2} & c_{3 n, 3 n-1} & c_{3 n, 3 n}
\end{array}\right]\left[\begin{array}{c}
f_{1 x} \\
f_{1 y} \\
f_{1 z} \\
\vdots \\
f_{n x} \\
f_{n y} \\
f_{n z}
\end{array}\right]
$$

Taking the first element of $\mathbf{U}^{\prime}$ as an example, $u_{1 x}$ is the displacement of node 1 in the $\mathrm{X}$ direction, the calculation is as follow:

$$
u_{1 x}=c_{11} f_{1 x}+c_{12} f_{1 y}+\cdots+c_{1,3 n-1} f_{n y}+c_{1,3 n} f_{n z}
$$

When node 1 is forced only in the $\mathrm{X}$ direction, i.e., $u_{1 x}=c_{11} f_{1 x}$, the stiffness of node 1 in the $\mathrm{X}$ direction is $k_{1 x}=f_{1 x} / u_{1 x}=1 / c_{11}$. Similarly, the stiffness of node 1 in the $\mathrm{Y}$ and $\mathrm{Z}$ direction is $k_{1 y}=1 / c_{22}$ and $k_{1 z}=1 / c_{33}$, respectively. Thus, the diagonal elements of the compliance matrix are used to calculate the node stiffness in the coordinate directions:

$$
\begin{aligned}
k_{n x} & =1 / c_{3 n-2,3 n-2} \\
k_{n y} & =1 / c_{3 n-1,3 n-1} \\
k_{n z} & =1 / c_{3 n, 3 n}
\end{aligned}
$$


Where $k_{n x}, k_{n y}$ and $k_{n z}$ are the stiffness of node $n$ in the $\mathrm{X}, \mathrm{Y}$ and $\mathrm{Z}$ axis, respectively. Then, the stiffness of the nodes in the group is screened out from all the node stiffness. Finally, after obtaining the stiffness of all nodes in their respective directions, the minimum stiffness of each machining region can be searched based on node-region affiliations.

\subsection{GNN-based minimum stiffness prediction of machining regions}

Graph neural network (GNN) is an artificial neural network that operates on graph domain. It has achieved remarkable results in various graph learning tasks in recent years [23]. Since the part model is represented as an attribute graph, GNN is the suitable model to predict the minimum stiffness of machining regions. The model input contains the part attribute graph and non-geometrical influence factors, including material properties, thickness, etc., while the model output is the minimum stiffness of all machining regions on the part.

\subsubsection{Sample features}

The considered sample features are composed of the influence factors of stiffness, including the non-geometrical factors and geometrical factors of the machining regions. The former contains the material properties, the area of constraint end, and minimum thickness of machining regions, etc., which can be directly vectorized as one-hot vectors. The latter includes the geometrical elements of the machining region and their topology information, i.e., the faces that make up the machining region and the connections among these faces, yet it is hard to directly represent them with vectors. For this problem, this paper represents the part model as an attribute graph and associates each machining region with a subgraph, which is based on our team's previous research work [24] [25]. Then, the vectorized representation of geometrical information will be obtained based on the GNN models during representation learning.

(1) The construction of part attribute graph. Gird nodes and their connections in finite element model can naturally be represented as a graph to describe the geometry, yet the large number of the nodes makes it difficult to process the graph efficiently. Thus, another graph is considered in this research, i.e., the graph constructed by the faces and edges of the part model. The 3D model of a part can be regarded as a closed entity surrounded by multiple faces, where the topology is the connections among those faces. Then, the part can be expressed as a graph $\mathcal{G}=(\mathcal{V}, \mathcal{E})$, where $\mathcal{V}$ is the set of vertexes, and $\mathcal{E}$ is the set of edges, corresponding to the faces and edges on the part model respectively, as shown in Fig. 5. In addition, considering that each face has geometrical attributes including normal vector, area, perimeter, etc. In this paper, 11 typical geometrical attributes are selected according to the correlation to the local stiffness, as listed in Table 1. These geometrical attributes compose the attribute vector $\boldsymbol{h}$, and all the attribute vectors form an attribute matrix $\mathbf{H}$. Therefore, the part attribute graph is expressed as $\mathcal{G}=(\mathcal{V}, \mathcal{E}, \mathbf{H})$.

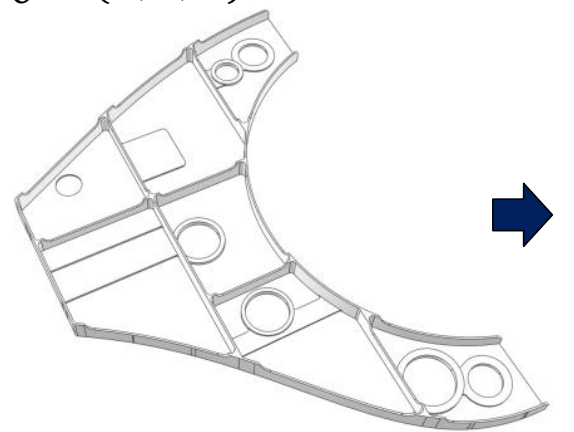

Part model

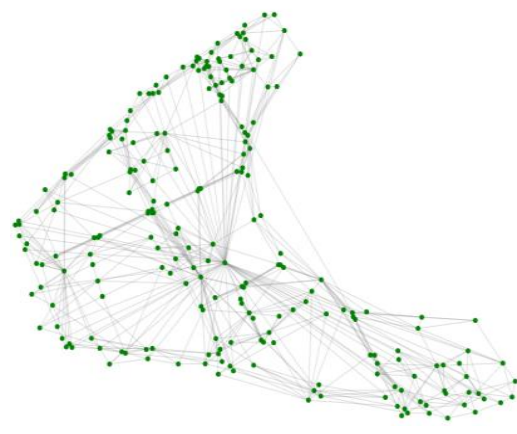

Graph

Fig. 5 The graph representation of the part model. 
Table 1 The selected geometrical attributes of each face in the part.

\begin{tabular}{llll}
\hline No. & Attributes & Data type & Dimension \\
\hline 1 & Normal vector & double & 3 \\
2 & Face type & integer & 3 \\
3 & Whether has inner loop & integer & 2 \\
4 & Area & double & 1 \\
5 & Perimeter & double & 1 \\
6 & The ratio of area to perimeter & double & 1 \\
7 & Min, max height from the bottom face & double & 2 \\
8 & The length of the shortest, longest edge & double & 2 \\
9 & Number of the edges & integer & 1 \\
10 & Number of convex, concave connections & integer & 2 \\
11 & Number of tangent, non-tangent connections & integer & 2 \\
\hline
\end{tabular}

After the part model is represented as an attribute graph, the machining regions correspond to subgraphs in the graph, and relation matrix $\mathbf{R} \in \mathbb{R}^{m \times t}$ is constructed to describe the correlation between each vertex and each subgraph, where $m$ is the number of vertexes, and $t$ is the number of machining regions. Let $f_{j}$ be a machining region, whose vertexes are represented as the set $\mathcal{F}_{j}$, then, the relation between vertex $v_{i}$ in $\mathcal{F}_{j}$ and the matrix element $r_{i j}$ in $\mathbf{R}$ is shown as follows,

$$
r_{i j}=\left\{\begin{array}{ll}
1 & v_{i} \in \mathcal{F}_{j} \\
0 & v_{i} \notin \mathcal{F}_{j}
\end{array} \quad(1 \leq i \leq m, 1 \leq j \leq t)\right.
$$

(2) Other influence factors. In addition to geometrical factors, some other nongeometrical influence factors are also important to the stiffness, including material properties (e.g., elastic modulus, Poisson's ratio and density), the area of constraint end, and minimum thickness of machining region, etc., as listed in Table 2 . These factors can be directly encoded as vectors.

Table 2 The other influence factors of stiffness in machining regions.

\begin{tabular}{llll}
\hline No. & Description & Data type & Dimension \\
\hline 1 & Elastic modulus & double & 1 \\
2 & Poisson's ratio & double & 1 \\
3 & Density & double & 1 \\
4 & The bottom thickness of the part & integer & 1 \\
5 & Area of constraint end of the part & double & 1 \\
6 & The volume of the part & double & 1 \\
7 & Max distance between machining region and the constraint end & double & 1 \\
8 & Min distance between machining region and the bottom face & double & 1 \\
9 & Min thickness of machining region & double & 1 \\
10 & Constraint condition of machining region & integer & 3 \\
\hline
\end{tabular}

\subsubsection{GNN architecture}

In this paper, four GNN models are built to predict the minimum stiffness of four types of machining regions. They have the same network architecture, including graph convolution layers, a machining region representation layer, and fully connected prediction layers. The input of GNN models contains the part attribute graph and the encoding vectors of non- 
geometrical influence factors, while the output is the minimum stiffness of machining regions. First, vertexes of the attribute graph are embedded as representation vectors in graph convolution layers. Then, in the machining region representation layer, each machining region is represented with the concatenation between the vertex representation and the vectorized non-geometrical influence factors. Finally, in fully connected prediction layers, further feature extraction is performed and processed to generate the prediction. The architecture of the GNN model is shown in Fig. 6.

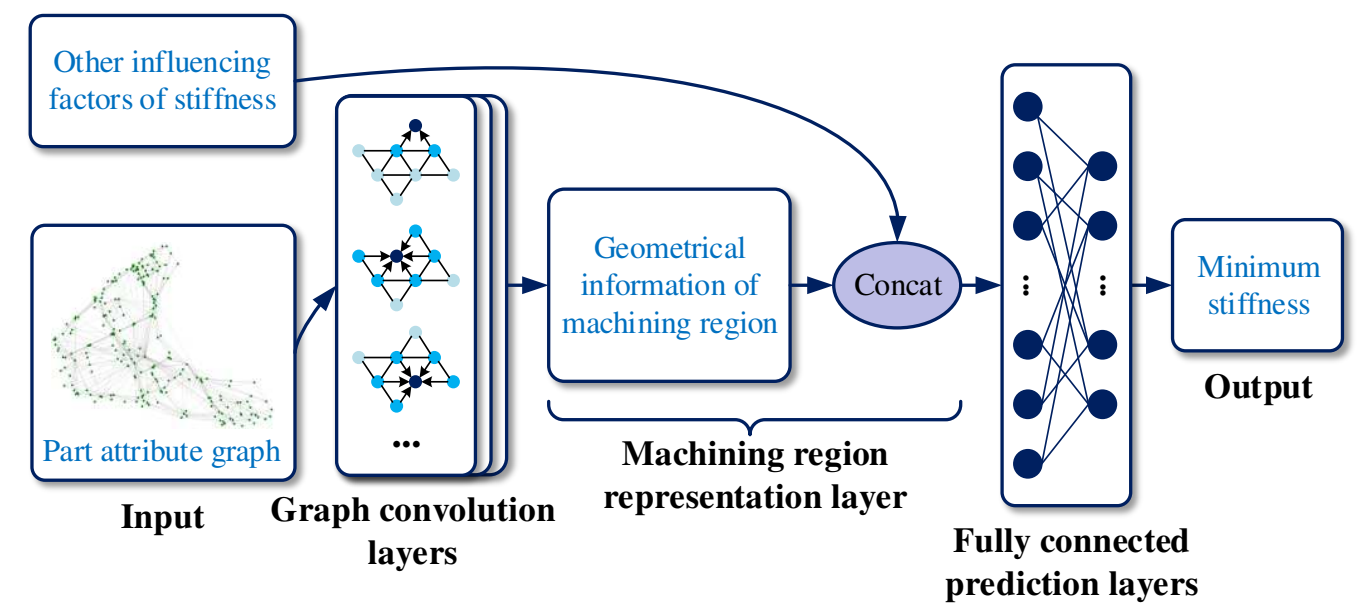

Fig. 6 The architecture of the GNN model in this paper.

(1) Graph convolution layers. For GNN, graph convolution is the main way to learn vertex representations. Existing graph convolution mainly follows the aggregation of vertex attributes, where each vertex absorbs the attributes of its neighbor vertexes. After multiple iterations of aggregation, the representation vector of each vertex contains both geometrical and topological information, as shown in Fig. 7. In this paper, graph attention mechanism is used to realize the aggregation of vertex attributes [26], as shown in formula (8):

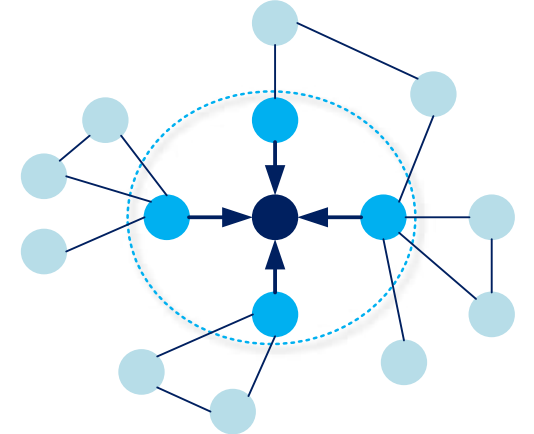

Fig. 7 The aggregation of vertex attributes in the graph. Each vertex absorbs the attributes of its neighbor in each iteration of aggregation, e.g., the dark blue vertex absorbs the attributes of the light blue vertexes.

$$
\boldsymbol{h}_{i}^{(l)}=A\left(\sum_{v_{j} \in \mathcal{N}_{i} \cup\left\{v_{i}\right\}} \alpha_{i j}^{(l-1)} \mathbf{W}^{(l-1)} \boldsymbol{h}_{i}^{(l-1)}\right)
$$

Where, $A(\cdot)$ is the activation function, $\mathcal{N}_{i}$ is the set of neighbor vertexes of vertex $v_{i}, \alpha_{i j}$ is the attention weight coefficient, which is based on node attributes, $\mathbf{W} \in \mathbb{R}^{F^{\prime} \times F}$ is a learnable weight matrix, and $\boldsymbol{h}_{i}^{(l)} \in \mathbb{R}^{F}(1 \leq i \leq m)$ is the representation vector of vertex $v_{i}$ at the $l$-th layer, where $m$ is the number of vertexes, $F$ the dimension of the attribute vector.

To make the learning of GNN more stable, multi-head is used, which performs $K$ aggregations simultaneously, and the results are combined together, as shown in formula (9): 


$$
\boldsymbol{h}_{i}^{(l)^{\prime}}=\bigcup_{k=1}^{K} \boldsymbol{h}_{i}^{(l)^{k}}
$$

Where, $U$ represents the concatenation operation except for the last aggregation, in which $U$ represents the summation operation.

Considering the size of the subgraph in this paper, it requires $2 \sim 3$ convolution layers to achieve proper representation learning.

(2) Machining region representation layer. The machining region representation layer is a single layer to represent the machining region, which contains two steps: The first step is to represent the geometrical information of the machining region, and the second step is to incorporate non-geometrical influence factors.

For the first step, since each machining region is correlated to a subgraph, summation is used to combine the representation of the vertexes contained in the subgraph to represent the geometrical information, as shown below:

$$
\boldsymbol{f}^{r}=\sum_{\boldsymbol{v}_{j} \in \mathcal{G}_{s u b}} \boldsymbol{h}_{j}^{\prime}
$$

Where, $\boldsymbol{f}^{r}$ is the geometrical information representation vector of the machining region, $\mathcal{G}_{\text {sub }}$ is a subgraph corresponding to the machining region, and $\boldsymbol{h}_{j}^{\prime}$ is the representation vector of vertex $v_{j}$.

For the second step, the representation of geometrical information is concatenated with the vectorized non-geometrical influence factors to completely represent a machining region:

$$
\boldsymbol{f}=\boldsymbol{f}^{r} \| \boldsymbol{f}^{\text {non }}
$$

Where, $\boldsymbol{f}$ and $\boldsymbol{f}^{\text {non }}$ are the complete representation vector and the vectorized nongeometrical influence factors of the machining region, and $\|$ is the concatenation operation.

(3) Fully connected prediction layers. The fully connected prediction layers are used to further extract features from the representation of machining regions, and then generate the stiffness predictions. In the constructed GNN model, there are 3 4 fully connected layers, each of which consists of a linear combination and a nonlinear activation except for the last prediction layer. Considering that the output minimum stiffness is continuous value, mean square error (MSE) is used as the loss function, as follow:

$$
\operatorname{loss}_{M S E}=\frac{1}{t} \sum_{i=1}^{t}\left(\hat{y}_{i}-y_{i}\right)^{2}
$$

Where, $\hat{y}_{i}$ and $y_{i}$ are the predicted value and label value respectively, $t$ is the total number of machining regions.

\section{Case Study}

To test the proposed model, a dataset is first built based on 36 aircraft structural parts including large, medium and small frame and beam parts, which are made from aluminum alloy. This dataset contains 36 attribute graphs, 3899 machining operations corresponding to 3899 machining regions, and the machining regions of bottom, sidewall, rib, and corner are 1094, 1072, 716 and 1017 respectively, as listed in Table 3. Then, four GNN models are established to predict the minimum stiffness of each type of the machining region. To validate the model performance, the predicted results on test set are compared with the calculation 
results from FEM to validate the predicting performance. The total computation time of both methods is compared to validate the computational efficiency.

Table 3 Details of the built dataset in this paper.

\begin{tabular}{llll|llll}
\hline Part No. & $\begin{array}{l}\text { Number of } \\
\text { faces }\end{array}$ & $\begin{array}{l}\text { Number of } \\
\text { edges }\end{array}$ & $\begin{array}{l}\text { Number of } \\
\text { machining } \\
\text { regions }\end{array}$ & Part No. & $\begin{array}{l}\text { Number of } \\
\text { faces }\end{array}$ & $\begin{array}{l}\text { Number of } \\
\text { edges }\end{array}$ & $\begin{array}{l}\text { Number of } \\
\text { machining } \\
\text { regions }\end{array}$ \\
\hline 1 & 324 & 940 & 137 & 19 & 265 & 774 & 117 \\
2 & 219 & 629 & 99 & 20 & 178 & 519 & 77 \\
3 & 357 & 1033 & 154 & 21 & 180 & 506 & 89 \\
4 & 192 & 559 & 66 & 22 & 164 & 459 & 76 \\
5 & 227 & 660 & 86 & 23 & 206 & 572 & 85 \\
6 & 233 & 675 & 100 & 24 & 175 & 493 & 88 \\
7 & 386 & 1128 & 188 & 25 & 207 & 597 & 93 \\
8 & 461 & 1294 & 185 & 26 & 151 & 437 & 66 \\
9 & 340 & 1003 & 131 & 27 & 276 & 773 & 117 \\
10 & 443 & 1237 & 192 & 28 & 105 & 305 & 35 \\
11 & 293 & 828 & 110 & 29 & 106 & 297 & 48 \\
12 & 481 & 1369 & 197 & 30 & 262 & 730 & 109 \\
13 & 338 & 969 & 127 & 31 & 238 & 697 & 96 \\
14 & 416 & 1218 & 184 & 32 & 96 & 275 & 33 \\
15 & 449 & 1310 & 187 & 33 & 104 & 290 & 46 \\
16 & 293 & 836 & 119 & 34 & 210 & 612 & 90 \\
17 & 343 & 996 & 142 & 35 & 159 & 464 & 58 \\
18 & 308 & 906 & 125 & 36 & 107 & 296 & 47 \\
\hline
\end{tabular}

\subsection{Data preparation}

The data preparation contains three aspects: the selection and acquisition of sample data, the selection and acquisition of label data, and data splitting. For the proposed model, the sample features include the geometrical information of the machining region and nongeometrical influence factors. The former is represented based on the part attribute graph, where each vertex contains 11 types of geometrical attributes that may affect the stiffness, as listed in Table 1. The later contains material properties, the area of constraint end, and the constraint condition of machining regions, etc., as listed in Table 2. Since the part material in the dataset is only aluminum alloy, the factors related to material properties are not considered. For data extraction, a data extraction tool was developed in CATIA based on Microsoft Visual Studio 2015 to effectively extract sample data.

In terms of label data, the minimum stiffness of all the machining regions on the part is calculated in ABAQUS, a widely used FEM software. The elastic modulus is 72000Mpa, the Poisson's ratio is 0.33 , the density is $2.82 \mathrm{e}-9$ tonne $/ \mathrm{mm}^{3}$, and the grid element type is C3D10.

For data splitting, 36 parts is divided into a training set with 34 parts, a validation set with 1 part and a test set with 1 part, the number of each type of machining region in each dataset is listed in Table 4.

Table 4 Details of the number of each type of machining region on different dataset.

\begin{tabular}{llllll}
\hline & GNN-bottom & GNN-sidewall & GNN-rib & GNN-corner & Total \\
\hline Training set & 1071 & 1049 & 694 & 1012 & 3816 \\
Validation set & 15 & 15 & 10 & 8 & 48 \\
Test set & 8 & 8 & 12 & 7 & 35 \\
\hline
\end{tabular}




\subsection{GNN development}

To predict the minimum stiffness of four types of machining regions, including bottom, sidewall, rib, and corner, four GNN models are developed based on TensorFlow 1.13.1 [27], a widely used deep learning framework proposed by Google. The input of the GNN models includes the part attribute graph and vectorized non-geometrical influence factors, while the output is the minimum stiffness of all machining regions on the part. The hyper-parameters of the GNN models are listed in Table 5.

Table 5 The hyper-parameters of the four GNN models.

\begin{tabular}{lllll}
\hline Parameters & $\begin{array}{l}\text { GNN- } \\
\text { bottom }\end{array}$ & $\begin{array}{l}\text { GNN- } \\
\text { sidewall }\end{array}$ & $\begin{array}{l}\text { GNN- } \\
\text { rib }\end{array}$ & $\begin{array}{l}\text { GNN- } \\
\text { corner }\end{array}$ \\
\hline Number of graph convolution layers & 2 & 3 & 3 & 3 \\
Number of units at the graph convolution layers & 30,30 & $20,20,20$ & $20,20,20$ & $20,20,20$ \\
Number of attention heads & 25,25 & $20,20,20$ & $20,20,20$ & $20,20,20$ \\
Number of machining region representation layer & 1 & 1 & 1 & 1 \\
Number of fully connected prediction layers & 3 & 4 & 4 & 3 \\
Number of units at the fully connected prediction layers & $25,15,8$ & $20,15,10,5$ & $20,15,10,5$ & $15,10,5$ \\
Learning rate & 0.0008 & 0.0006 & 0.0018 & 0.0015 \\
\hline
\end{tabular}

\subsection{Validation}

\subsubsection{Predicting performance}

Before analyzing the predicting performance, loss curves are used to observe the learning process of the GNN models, as shown in Fig. 8. The overall trends of these four loss curves are very similar. The loss curve of each GNN model drops rapidly at the beginning, then the decreasing trend gradually slows down, and after 1200 training steps, the loss curve tends to be stable. Thus, the overall training trend is regular, each model is trained rapidly first, then slowly, and finally the model is stable.
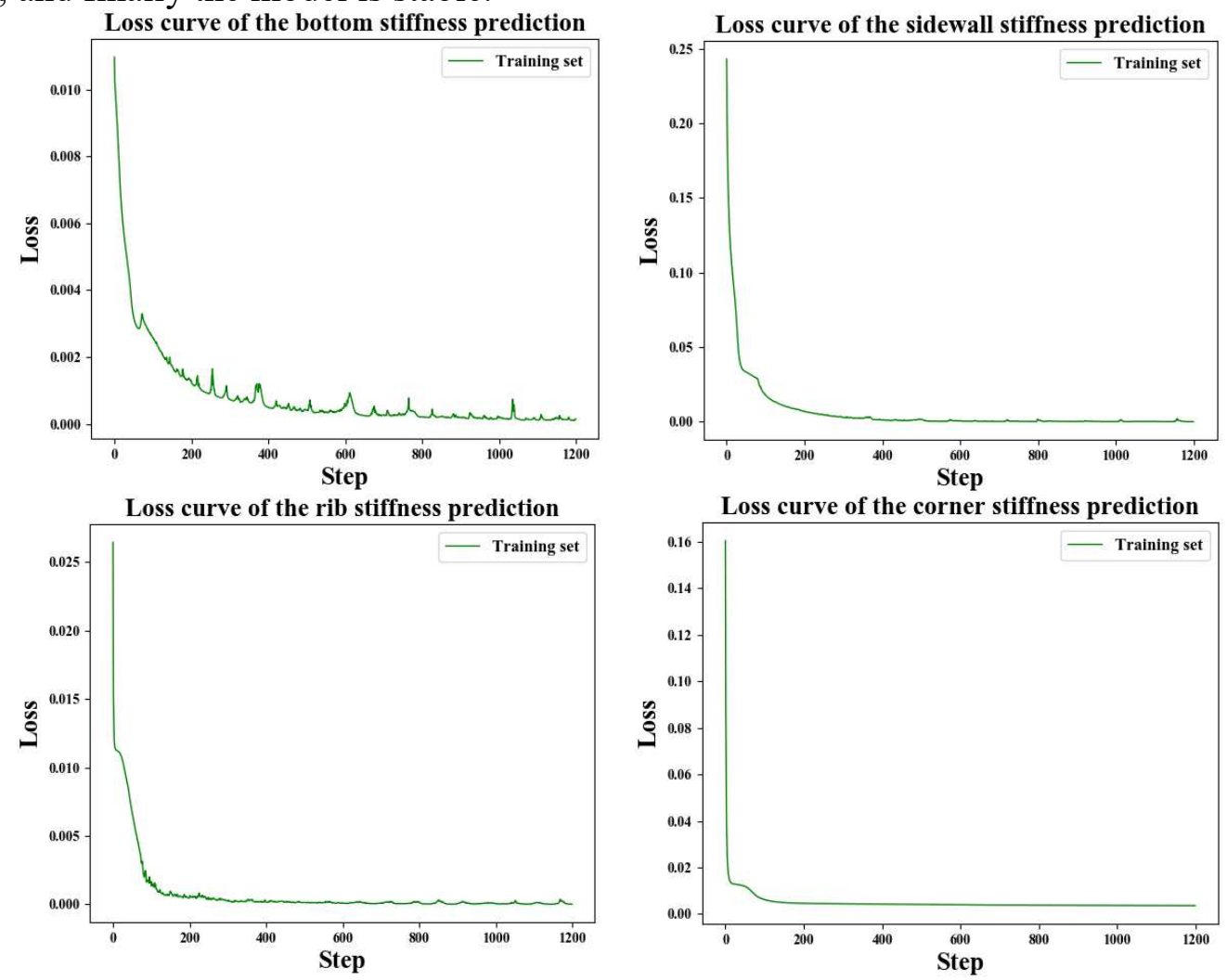

Fig. 8 The loss curves of the proposed models on training set. 
To analyze the predicting performance, the model prediction on validation set and test set is compared with the FEM calculation results, where the FEM calculation results are taken as the baseline, and the average percentage errors (APE) is used as the metric, as follow,

$$
\mathrm{APE}=\frac{1}{t} \sum_{i=1}^{t} \frac{\left|\hat{k}_{i}-k_{i}\right|}{k_{i}} \times 100 \%
$$

Where $\hat{k}_{i}$ and $k_{i}$ are the predicted value of the proposed model and calculation value of FEM respectively, $t$ is the total number of machining regions. The results are shown in Table 6 . The model performance on validation set and test set is very close, which indicates that the generalization performance of the model is stable. Furthermore, each metric is less than $10 \%$. According to the engineering experience, the predicting result can be used in planning machining parameters.

Table 6 The average percentage errors of four GNN models on validation set and test set.

\begin{tabular}{lll}
\hline Prediction model & Validation set & Test set \\
\hline GNN-bottom & $8.693 \%$ & $6.717 \%$ \\
GNN-sidewall & $6.314 \%$ & $7.367 \%$ \\
GNN-rib & $6.689 \%$ & $7.432 \%$ \\
GNN-corner & $7.595 \%$ & $5.962 \%$ \\
\hline
\end{tabular}

\subsubsection{Computational efficiency}

To validate the computational efficiency of the proposed method, taking a test part as an example, the total computation time of the proposed method and FEM based calculation is compared. The part dimension is $1534 \mathrm{~mm} \times 208 \mathrm{~mm}$, the number of the grid elements in FEM is 12920 , and the number of nodes is 26602 .

The steps of FEM and the time spent at each step are as follows. First, preparations were made. The minimum stiffness directions of all nodes were calculated, and 5 coordinate systems were built to divide the nodes into 5 groups based on the calculated directions, which took $4 \mathrm{~min}$. Then, for each group of nodes, the stiffness calculation contained 5 steps: (1) All element stiffness matrices in the global coordinate system were calculated once in total, which took 3.5min. (2) Coordinate system transformation was used to obtain the element stiffness matrices in the new coordinate system corresponding to each group. Since there was a group whose coordinate system was the global coordinate system, it required a total of 4 calculations, which took $4 \times 0.5 \mathrm{~min}$. (3) The global stiffness matrix was combined based on the calculated element stiffness matrices, which took $5 \times 2.5 \mathrm{~min}$. (4) The global compliance matrix was calculated by reversing the global stiffness matrix, and the stiffness of all the nodes was further obtained, which took $5 \times 33 \mathrm{~min}$. (5) The stiffness of the nodes in each group was screened out, because each group took less than $0.1 \mathrm{~s}$, the time spent can be ignored. After obtaining the stiffness of all nodes in the respective directions, the minimum stiffness of each machining region was searched in $0.1 \mathrm{~s}$, which can also be ignored. Thus, the FEM calculation took $187 \mathrm{~min}$ in total.

The proposed data driven method contained 3 steps: (1) Data preparation, including sample feature and label data acquisition, the former was fast, yet the latter was time consuming, which required $34 \mathrm{FEM}$ calculation corresponding to 34 parts on the training set. (2) Model training, which spent $10.5 \mathrm{~h}, 12 \mathrm{~h}, 11.5 \mathrm{~h}, 9.5 \mathrm{~h}$ for four GNN models, respectively. (3) The application of the trained models. The four trained GNN models took $23 \mathrm{~s}, 28 \mathrm{~s}, 25 \mathrm{~s}$, $26 \mathrm{~s}$ in predicting, respectively, i.e., 102s in total. Although the data acquisition and the model training require much time, once the training is completed, the proposed model can efficiently calculate the stiffness of any other similar parts, which can greatly reduce the time compared 
with FEM.

\section{Conclusion and Future Work}

Large thin-walled structural parts are easy to deform during machining if improper cutting parameters are selected, thus local stiffness has to be seriously considered during machining parameter planning. However, existing methods for calculating local stiffness of the part are either inaccurate or time-consuming. To address this issue, this paper proposes a data-driven method for local stiffness prediction of aircraft structural parts. The main contributions of this paper are as follows:

(1) The machining regions of aircraft structural parts are divided into bottom, sidewall, rib and corner, and their minimum stiffness is defined and further calculated based on FEM.

(2) The minimum stiffness prediction of a machining region is converted to a graph learning task by representing the part geometry as an attribute graph.

(3) Four GNN models are designed and trained to map the attribute graphs of the four types of machining regions to their minimum stiffness, respectively.

The proposed data-driven method can efficiently predict the minimum stiffness of all the machining regions of aircraft structural parts, and the model training is end-to-end, which is convenient once the data is sufficient. In terms of model performance, the predicted results are compared with the calculation results from FEM in case study, and taking FEM results as the baseline, the average percentage errors of the predicting stiffness corresponding to four types of machining regions are $6.717 \%, 7.367 \%, 7.432 \%$ and $5.962 \%$ respectively, which can meet the requirements in planning machining parameters according to the engineering experience. Besides, in terms of computational efficiency, once the model training is completed, the proposed model can efficiently calculate the stiffness of any other similar parts, which can greatly reduce the time compared with FEM. In the future work, the proposed method will be combined with the optimization of machining parameters for aircraft structural parts to improve the machining efficiency while ensuring the machining quality.

Funding This research was funded by the National Natural Science Foundation of China (Grant No. 51925505 and U21B2081).

\section{Declarations}

Ethics approval and consent to participate Not applicable.

Consent for publication Approved.

Competing interests The authors declare no competing interests.

\section{References}

[1] Herranz S, Campa FJ et al (2005) The milling of airframe components with low rigidity: a general approach to avoid static and dynamic problems[J]. Proc inst Mech Eng, Part B: J Eng Manuf 219(11):789-802. https://doi.org/10.1243/095440505x32742

[2] Yang Y, Li M et al (2014) Comparison and analysis of main effect elements of machining distortion for aluminum alloy and titanium alloy aircraft monolithic component[J]. Int J Adv Manuf Technol 70(9):1803-1811. https://doi.org/10.1007/s00170-013-5431-x

[3] Zhao W, Li Y, Liu C et al (2016) A cutting parameter optimization method based on dynamic machining features for complex structural parts[C]. 2016 IEEE 20th International Conference on Computer Supported Cooperative Work in Design (CSCWD). IEEE 85-90. https://doi.org/10.1109/CSCWD.2016.7565968

[4] Jia Z, Lu X, Gu H et al (2021) Deflection prediction of micro-milling Inconel 718 thin-walled parts[J]. J Mater Process Technol 291:117003. https://doi.org/10.1016/j.jmatprotec.2020.117003

[5] Ma J, He G, Liu Z et al (2018) Instantaneous cutting-amount planning for machining deformation homogenization 
based on position-dependent rigidity of thin-walled surface parts[J]. J Manuf Process 34:401-411. https://doi.org/10.1016/j.jmapro.2018.05.027

[6] Wang X, Li Z, Bi Q et al (2019) An accelerated convergence approach for real-time deformation compensation in large thin-walled parts machining[J]. Int J Mach Tools Manuf 142:98-106. https://doi.org/10.1016/j.ijmachtools.2018.12.004

[7] Qian C, Ye W (2021) Accelerating gradient-based topology optimization design with dual-model artificial neural networks[J]. Struct Multidisciplinary Optim 63:1687-1707. https://doi.org/10.1007/s00158-020-02770-6

[8] Tang A, Liu Z (2008) Deformations of thin-walled plate due to static end milling force[J]. J Mater Process Technol 206(1-3):345-351. https://doi.org/10.1016/j.jmatprotec.2007.12.089

[9] Wu Q, Li D, Ren L et al (2016) Detecting milling deformation in 7075 aluminum alloy thin-walled plates using finite difference method[J]. Int J Adv Manuf Technol 85(5-8):1291-1302. https://doi.org/10.1007/s00170-015-8012-3

[10] Gao H, Zhang Y, Wu Q et al (2018) Investigation on influences of initial residual stress on thin-walled part machining deformation based on a semi-analytical model[J]. J Mater Process Technol 262:437-448. https://doi.org/10.1016/j.jmatprotec.2018.04.009

[11] Liu C, Li Y, Zhou X et al (2015) Interim feature-based cutting parameter optimization for aircraft structural parts[J]. Int J Adv Manuf Technol 77(1-4):663-676. https://doi.org/10.1007/s00170-014-6487-y

[12] Zhang T, Chen W (2010) Rigidity expression model research of barrel thin-walled parts[J]. Aeronaut Manuf Technol 9:71-73. https://doi.org/10.3969/j.issn.1671-833X.2010.09.015

[13] Wang GP (2013) Research on the pressure-relift characteristics of $0.6 \mathrm{MN}$ pump-controlled press system[D]. Yanshan University, 2013.

[14] Wan M, Zhang WH, Qiu KP et al (2005) Numerical prediction of static form errors in peripheral milling of thin-walled workpieces with irregular meshes[J]. J Manuf Sci Eng 127(1):13-22. https://doi.org/10.1115/1.1828055

[15] Liu S, Shao X, Ge X et al (2017) Simulation of the deformation caused by the machining cutting force on thin-walled deep cavity parts[J]. Int J Adv Manuf Technol 92:3503-3517. https://doi.org/10.1007/s00170-017-0383-1

[16] Smith S, Wilhelm R et al (2012) Sacrificial structure preforms for thin part machining[J]. CIRP Ann Manuf Technol 61(1):379-382. https://doi.org/10.1016/j.cirp.2012.03.142

[17] Huang X, Sun J et al (2017) Mathematical modeling of aeronautical monolithic component machining distortion based on stiffness and residual stress evolvement[J]. Chin J Mech Eng 53(9):201-208. https://doi.org/10.3901/JME.2017.09.201

[18] Nestor V. Queipo, Raphael T. Haftka, Wei Shyy et al (2005) Surrogate-based analysis and optimization[J]. Prog Aerosp Sci 41:1-28. https://doi.org/10.1016/j.paerosci.2005.02.001

[19] Wang G, Shan S (2007) Review of metamodeling techniques in support of engineering design optimization[J]. J Mech Des 129(4):370-380. https://doi.org/10.1115/1.2429697

[20] Sun D, Luo P et al (1997) Neurocomputing method of finite element stiffness matrix[J]. J Northeastern Univ 18(4):431434.

[21] Jia G, Yu Y et al (2020) Solving finite element stiffness matrix based on convolutional neural network[J]. J Beijing Univ Aeronaut Astronaut 46(3):481-487. https://doi.org/10.13700/j.bh.1001-5965.2019.0134

[22] White D, Arrighi W, Kudo J et al (2019) Multiscale topology optimization using neural network surrogate models[J]. Comput Methods Appl Mech Eng, 346:1118-1135. https://doi.org/10.1016/j.cma.2018.09.007

[23] Zhou J, Cui G, Hu S et al (2020) Graph neural networks: a review of methods and applications[J]. AI Open 1:57-81. https://doi.org/10.1016/j.aiopen.2021.01.001

[24] Deng T, Li Y, Liu X et al (2021) A data-driven parameter planning method for structural parts NC machining[J]. Robot Com-Integr Manuf 68:102080. https://doi.org/10.1016/j.rcim.2020.102080

[25] Deng T, Li Y, Chen J et al (2021) Informed machine learning-based machining parameter planning for aircraft structural parts[J]. Int J Adv Manuf Technol 117:3563-3575. https://doi.org/10.1007/s00170-021-07861-2

[26] Veličković P, Cucurull G, Casanova A et al (2017) Graph attention networks[J]. arXiv preprint arXiv:1710.10903.

[27] Abadi M, Barham P, Chen J et al (2016) Tensorflow: a system for large-scale machine learning[C]. 12th \{USENIX\} Symposium on Operating Systems Design and Implementation (\{OSDI\} 16). 2016:265-283. 